\title{
Management of severe hypertriglyceridemia due to lipoprotein lipase deficiency in children
}

\section{Sarah W Y Poon, Karen K Y Leung and Joanna Y L Tung}

Department of Paediatrics and Adolescent Medicine, Queen Mary Hospital, The University of Hong Kong, Hong Kong, Hong Kong
Correspondence should be addressed to J Y L Tung

Email

tungylj@hku.hk

\section{Summary}

Severe hypertriglyceridemia is an endocrine emergency and is associated with acute pancreatitis and hyperviscosity syndrome. We describe an infant with lipoprotein lipase deficiency with severe hypertriglyceridemia who presented with acute pancreatitis. She was managed acutely with fasting and intravenous insulin infusion, followed by low-fat diet with no pharmacological agent. Subsequent follow-up until the age of 5 years showed satisfactory lipid profile and she has normal growth and development.

\section{Learning points:}

- Hypertriglyceridemia-induced acute pancreatitis has significant morbidity and mortality, and prompt treatment is imperative.

- When no secondary causes are readily identified, genetic evaluation should be pursued in hypertriglyceridemia in children.

- Intravenous insulin is a safe and effective acute treatment for hypertriglyceridemia in children, even in infants.

- Long-term management with dietary modifications alone could be effective for primary hypertriglyceridemia due to lipoprotein lipase deficiency, at least in early childhood phase.

\section{Background}

Hypertriglyceridemia is defined as fasting plasma triglyceride (TG) level above an age and gender - adjusted 95th percentile. Monogenic hypertriglyceridemia include genetic defects in triglyceride metabolism, whereas polygenic hypertriglyceridemia are often due to interplay between genetic, hormonal and environmental factors including uncontrolled diabetes, obesity, metabolic syndrome and medications in the pediatric age group. Severe hypertriglyceridemia, defined as triglyceride concentration of greater than $1000 \mathrm{mg} / \mathrm{dL}$, can present with pancreatitis in the first decade of life and should prompt consideration of monogenic hypertriglyceridemia.
The clinical severity and rate of complications with hypertriglyceridemic pancreatitis are generally higher, thus appropriate treatment and prevention of disease recurrence is essential. As such, there is no consensus on the acute and long-term management of severe hypertriglyceridemia in the pediatric population. Our case highlights the use of insulin as an adjunct to lower the TG, followed by medium-chain triglyceride (MCT)based diet, and subsequently, strict dietary fat restrictions as long-term management in a young infant with familial chylomicronemia syndrome (FCS) due to lipoprotein lipase (LPL) deficiency. 


\section{Case presentation}

A 38-day-old Chinese girl presented to the emergency department for vomiting, irritability and suspected seizure. She was born full term with a birth weight of $2.8 \mathrm{~kg}$ with uneventful postnatal course. She was on mixed feeding (breast milk supplemented with normal formula) and was growing along the 25th centile. Her parents are non-consanguineous Chinese and her father has hypertriglyceridemia with triglyceride (TG) level at $250-300 \mathrm{mg} / \mathrm{dL}$ and diabetes mellitus requiring medication since the age of 40 years. There is otherwise no family history of dyslipidemia or pancreatitis.

On admission, she was noted to have hepatosplenomegaly. Physical examination was unremarkable otherwise and there were no skin lesions or abnormal body fat distribution. During venesection, milky serum was noted (Fig. 1A and B).

\section{Investigation}

TG levels were found to be $>13,290 \mathrm{mg} / \mathrm{dL}$ with the total cholesterol level at $919 \mathrm{mg} / \mathrm{dL}$. Serum lipase was elevated at $2534 \mu / \mathrm{L}$ (normal $23-300 \mu / \mathrm{L}$ ), while amylase level was normal. Serum sodium was $129 \mathrm{mEq} / \mathrm{L}$, potassium $5.1 \mathrm{mEq} / \mathrm{L}$, adjusted calcium $8.12 \mathrm{mg} / \mathrm{dL}$ and bicarbonate $18 \mathrm{mEq} / \mathrm{L}$. Computed tomography of the abdomen showed indistinct pancreatic head with loss of glandular structure, while the uncinated process, pancreatic body and tail displayed homogenous enhancement, suggestive of pancreatitis.

Agarose gel electrophoresis showed a dense chylomicron band, while the intensity of the very lowdensity lipoprotein (VLDL) band was mildly increased,
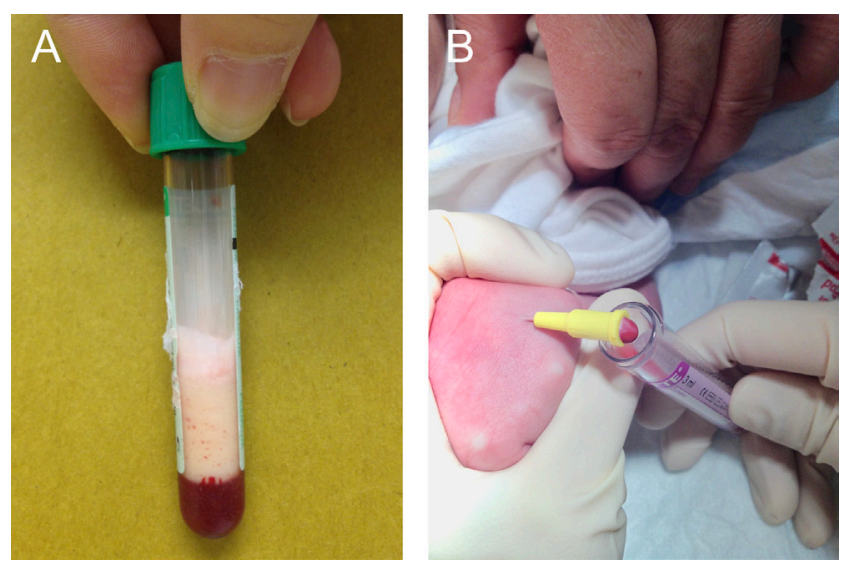

Figure 1

( $A$ and $B$ ) Milky serum noted on blood taking. compatible with type I hyperlipidemia with LPL deficiency. Subsequent genetic testing confirmed two heterozygous LPL variants, p.Cys54 (p.Cys27 in mature protein) (C27X) and p.Leu279Val (L279V), which have been reported to be pathogenic mutations $(1,2)$. Parents declined genetic studies due to personal reasons.

\section{Treatment}

She was kept fasted and started on intravenous dextrose. TG level dropped to $9232 \mathrm{mg} / \mathrm{dL}$ after $11 \mathrm{~h}$ of fasting. Intravenous insulin infusion was then started at $0.05 \mathrm{unit} / \mathrm{kg} / \mathrm{h}$ around $12 \mathrm{~h}$ after admission. TG level decreased slowly to $106 \mathrm{mg} / \mathrm{dL}$ after $84 \mathrm{~h}$ before the infusion was stopped. Octreotide infusion at $0.3 \mu \mathrm{g} / \mathrm{kg} / \mathrm{h}$ was started for acute pancreatitis and serum lipase gradually normalized (Fig. 2).

Feeding was resumed after 1 week of fasting with Monogen, a low-fat, high-MCT infant milk formula (84\% MCT, 16\% long-chain triglyceride) containing $12.9 \mathrm{~g}$ fat per $100 \mathrm{~g}$, with total fat intake of $9-12 \mathrm{~g}$ per day.

\section{Outcome and follow-up}

She recovered uneventfully and weaning diet was started at 6 months of age with low-fat diet (fat calorie 15\% of total calories). Until the age of 5 years, triglyceride level was maintained at $195-265 \mathrm{mg} / \mathrm{dL}$, while high-density lipoprotein cholesterol remained low at $11.5-19 \mathrm{mg} / \mathrm{dL}$. She was later found to have mild vitamin D deficiency and was put on calciferol supplement, while vitamin B12, folate and zinc levels were satisfactory and clotting profile was normal.

There were no further episodes of acute pancreatitis. Her growth is along the 25th centile and she has normal development.

\section{Discussion}

We describe an infant who had acute pancreatitis resulting from hypertriglyceridemia due to LPL deficiency, the most common cause of FCS. Other mutations include apolipoprotein C2 (APOC2), apolipoprotein A5 (APOA5), glycosylphosphatidylinositol-anchored high-density lipoprotein-binding protein 1 (GP1HBP1) and lipase maturation factor 1 (LMF1), which are important co-factors of LPL. LPL hydrolyzes triglyceride of chylomicrons and VLDLs after fat intake. Mutation in genes encoding LPL and its co-factors thus result in impaired lipolytic cascade and accumulation of chylomicrons in the plasma. 


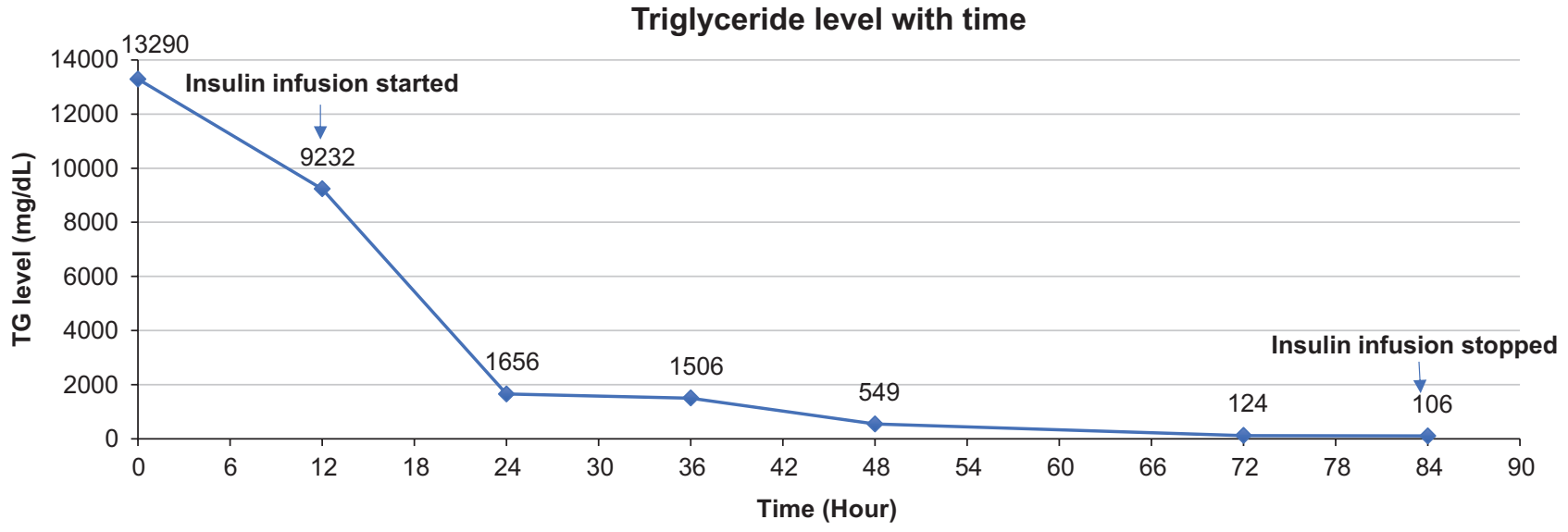

Figure 2

Trend of triglyceride level with time after treatment initiated.

As in our patient, affected individuals typically present with lipemia plasma during blood sampling. While abdominal pain is suggestive of pancreatitis in older children, presentation during infancy is nonspecific and heterogeneous. Other physical findings may include eruptive xanthomas, lipemic retinitis and hepatosplenomegaly, but it may not be present in all cases and could be non-specific. Due to interference by markedly elevated plasma triglyceride level, amylase level may be spuriously normal even with pancreatitis. Thus, measurement of serum lipase level is essential in this situation.

Management of hypertriglyceridemia in children is challenging as there is lack of established guideline.

As such, we aim to review the acute and long-term management in children.

\section{Acute management}

\section{Fasting}

Since chylomicrons are produced in the small intestine and are dependent on dietary fat for formation, fasting results in robust decline in TG levels and allows gradual clearance of existing chylomicrons. Likewise, our patient had significant drop in TG level after $11 \mathrm{~h}$ of fasting. Adequate hydration should be maintained with intravenous fluid. No fat diet, such as glucose water, can be introduced when TG level reaches $<1000 \mathrm{mg} / \mathrm{dL}$, and diet containing less than $10-15 \%$ fat can then be gradually resumed.

\section{Insulin}

Insulin is a rapid and potent activator of LPL through stimulation of its synthesis, thereby accelerates TG degradation into fatty acid and glycerol and facilitates storage in adipocytes (3). Therefore, insulin would only be useful if there is residual LPL activity. In a number of case reports in adults, insulin has been shown to be effective in reducing TG levels. However, case report on insulin use in patients with FCS, in particular due to LPL deficiency, is limited. While our case demonstrated sustained decline in TG level with insulin infusion, one cannot ascertain if similar effect could be achieved by fasting alone. Indeed, functional data related to both L279V and C27X mutations are limited. Theoretically, C27X creates a premature termination in the translation of the LPL peptide, and the very short truncated peptide with only 26 amino acids is expected to carry no catalytic function (2). Therefore, insulin may have only contributed modest effect in our case. However, at the initial presentation, such information was not available. The clinical decision to initiate insulin infusion was mainly based on the balance between the potential risks and benefits. Intravenous insulin given as continuous infusion, at $0.1-0.3$ units $/ \mathrm{kg} / \mathrm{h}$, allows easier titration of doses compared to subcutaneous boluses. The major risk with intravenous insulin, especially in infants, is hypoglycemia. Concomitant dextrose infusion, along the same intravenous line as insulin infusion, is important to maintain euglycemia and to stimulate endogenous insulin secretion. Blood glucose should be monitored closely during insulin infusion. In our case, we started insulin infusion 0.05 units $/ \mathrm{kg} / \mathrm{h}$ in view of her young age. Together with fasting, a rapid decline in TG level was observed with no side effects such as hypoglycemia noted. It is thus an effective, simple and safe adjunct treatment strategy for pediatric patients with severe hypertriglyceridemia. 


\section{Plasmapheresis and exchange transfusion}

Plasmapheresis allows direct removal of lipoprotein particles, decreases plasma viscosity and eliminates plasma proteases associated with pancreatitis. Pediatric case report is limited except for its use in a 10-year-old patient with diabetic ketoacidosis, hypertriglyceridemia and acute pancreatitis, in which rapid lowering of TG was observed (4). The use of plasmapheresis, however, is limited due to limited availability of the procedure and technical difficulty especially in young children. Moreover, in monogenic hypertriglyceridemia, rapid accumulation of TG can occur after plasmapheresis if dietary fat is not restricted. Whole blood exchange transfusion may be considered when plasmapheresis is not feasible, and excellent response has been reported in a young infant with severe hypertriglyceridemia with multi-organ failure (5).

\section{Heparin}

Heparin stimulates release of the endothelial LPL into the circulation, but as the increase in LPL activity is transient and followed by a rapid depletion in LPL plasma store, its use is controversial and not routinely recommended (6). Heparin may also not be effective if there is no residual LPL activity. Furthermore, there is risk of hemorrhage into the pancreas in patients with pancreatic necrosis.

\section{Long-term management}

Acute pancreatitis, which can be consequent to severe TG of $>1000 \mathrm{mg} / \mathrm{dL}$, is the most worrisome complication of hypertriglyceridemia in children. Long-term management should therefore aim to keep TG concentration less than $500 \mathrm{mg} / \mathrm{dL}$, as post-prandial TG concentration can increase exponentially.

\section{Diet}

The best long-term management in hypertriglyceridemia in FCS aims to reduce chylomicron formation by using low-fat diet that restricts fat intake to $10-15 \%$ of the total caloric consumption. In infants, formula high in MCT can be used to provide dietary calorie. MCTs are hydrolyzed to MCT fatty acids in the intestinal mucosa, which travel via the portal vein to the liver. They do not require bile acids and pancreatic lipase for absorption and can provide calories without being incorporated into chylomicrons, independent of the LPL pathway. Apart from ensuring compliance, monitoring for vitamins and micronutrients deficiency, regular anthropometric measurements should also be done for children on fat-restricted diet. Our patient tolerated Monogen well, and a low-fat weaning diet was introduced at 6 months. With strict diet control, her TG levels remained stable with satisfactory growth and development. However, compliance to low-fat diet could be challenging for school children, and support from dietician is required to maintain low-fat intake targets. Importance of avoidance of alcohol, a secondary factor that can contribute to hypertriglyceridemia, should be emphasized to teenage patients.

\section{Pharmacological treatment}

Currently, there are no Food and Drug Administration (FDA)-approved medications for FCS. Traditional lipidlowering drugs have limited success as their mechanism of action depends on a functional LPL lipolytic pathway (7). Fibrates reduce hepatic-derived TG-rich VLDL particles but not intestinally derived chylomicrons and may only be effective in those with residual LPL function. Its effectiveness in our case may be limited as LPL function is likely to be largely compromised, as in many patients with FCS. The triglyceride-lowering effects of niacin in FCS has also not been specifically evaluated. Data on use of omega-3 fatty acids in FCS are limited and they are unlikely to be effective as the primary problem is defective lipolysis.

\section{Gene therapy}

Alipogene tiparvovec was developed as a potential treatment for LPL deficiency. It is an adeno-associated virus type I (AAV1) gene therapy which delivers transgenic LPL to muscle in patients lacking the functional enzyme. Pre-clinical studies suggested that it results in muscle expression of LPL and a transient reduction in triglyceride levels (8). Although reported to reduce incidence of pancreatitis, sustained reduction in TG was not demonstrated, and there is no reported case in children. The therapy was licensed by the European Medicines Evaluation Agency but was withdrawn in 2017 due to poor commercial prospects and uncertainties about reimbursement.

\section{Antisense oligonucleotide}

Apolipoprotein C-III (APOC3) inhibits LPL-mediated hydrolysis of triglyceride-rich lipoproteins and adversely affect receptor-mediated hepatic uptake of remnants of triglyceride-rich lipoproteins. Volanesorsen is a secondgeneration antisense oligonucleotide that inhibits translation of Apo-CIII mRNA and is shown to lower plasma levels of Apo-CIII and triglycerides in patients 
with FCS (9). It is shown to be effective in FCS caused by LPL-null mutations with $<5 \%$ residual function of LPL (10), and thus, may be useful in our patient.

\section{Conclusion}

Our case highlights the acute and long-term management of a young infant with hypertriglyceridemia due to LPL deficiency. While consensus is lacking, fasting and insulin seem to be a safe, non-invasive and effective treatment strategy in the acute setting. Given the limited efficacy of pharmacological therapy, the mainstay of long-term management remains strict dietary fat restriction at present. With increased understanding of the disease, novel agents have been developed and may play a role in future management of FCS.

\section{Declaration of interest}

The authors declare that there is no conflict of interest that could be perceived as prejudicing the impartiality of this case report.

\section{Funding}

This work did not receive any specific grant from any funding agency in the public, commercial or not-for-profit sector.

\section{Patient consent}

Written informed consent has been obtained from the patient's guardian.

\section{Author contribution statement}

Joanna $Y$ L Tung is the main endocrinologist physician who followed the patients and she reviewed and edited the manuscript.
Sarah W Y Poon and Karen K Y Leung conducted the literature review and manuscript construction.

\section{References}

1 Chen TZ, Xie SL, Jin R \& Huang ZM. A novel lipoprotein lipase gene missense mutation in Chinese patients with severe hypertriglyceridemia and pancreatitis. Lipids in Health and Disease 201413 52. (https://doi.org/10.1186/1476-511X-13-52)

2 Chan AO, But W, Lau GT, Tse W \& Shek C. A novel nonsense mutation in the LPL gene in a Chinese neonate with hypertriglyceridemia. Clinica Chimica Acta 2006368 120-124. (https://doi.org/10.1016/j.cca.2005.12.020)

3 Flier JS, Underhill LH \& Eckel RH. Lipoprotein lipase. New England Journal of Medicine 1989320 1060-1068. (https://doi.org/10.1056/ NEJM198904203201607)

4 Lutfi R, Huang J \& Wong HR. Plasmapheresis to treat hypertriglyceridemia in a child with diabetic ketoacidosis and pancreatitis. Pediatrics 2012129 e195-e198. (https://doi. org/10.1542/peds.2011-0217)

5 Ghoor S, Berlyn P \& Brey N. Exchange transfusions for extreme hypertriglyceridemia in a 7-week-old infant with multi-organ failure. Journal of Clinical Lipidology 201812 243-245. (https://doi. org/10.1016/j.jacl.2017.10.018)

6 Näsström B, Olivecrona G, Olivecrona T \& Stegmayr BG. Lipoprotein lipase during continuous heparin infusion: tissue stores become partially depleted. Journal of Laboratory and Clinical Medicine 2001 138 206-213. (https://doi.org/10.1067/mlc.2001.117666)

7 Brahm AJ \& Hegele RA. Chylomicronaemia - current diagnosis and future therapies. Nature Reviews: Endocrinology 201511 352-362. (https://doi.org/10.1038/nrendo.2015.26)

8 Gaudet D, Méthot J \& Kastelein J. Gene therapy for lipoprotein lipase deficiency. Current Opinion in Lipidology 201223 310-320. (https:// doi.org/10.1097/MOL.0b013e3283555a7e)

9 Gaudet D, Alexander VJ, Baker BF, Brisson D, Tremblay K, Singleton W, Geary RS, Hughes SG, Viney NJ, Grahan MJ, et al. Antisense inhibition of apolipoprotein C-III in patients with hypertriglyceridemia. New England Journal of Medicine 2015373 438-447. (https://doi.org/10.1056/NEJMoa1400283)

10 Gaudet D, Brisson D, Tremblay K, Alexander VJ, Singleton W, Hughes SG, Geary RS, Baker BF, Graham MJ, Crooke RM, et al. Targeting APOC3 in the familial chylomicronemia syndrome. New England Journal of Medicine 2014371 2200-2206. (https://doi. org/10.1056/NEJMoa1400284)

Received in final form 7 June 2019

Accepted 2 July 2019 